\title{
Gestión de los recursos domiciliarios desde la utilización de software y hardware libre
}

\author{
Home Utilities Management with Free Software and Hardware
}

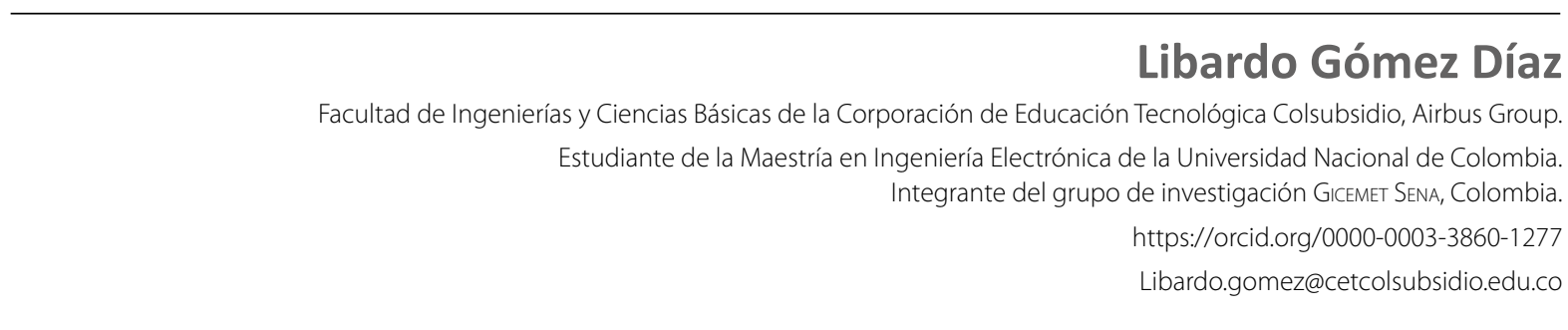

Fecha de recepción: 31 de marzo del 2019

Fecha de aceptación: 24 de mayo del 2019

Sugerencia de citación: Gómez-Díaz, L. (2019). Gestión de los recursos domiciliarios desde la utilización de software y hardware libre. Mutis, 9(1), 40-49 . doi: 10.21789/22561498.1461

\section{RESUMEN}

Este artículo evidencia la creación y utilización de un sistema de monitoreo y gestión de recursos domiciliarios para agua y energía en el hogar que permite la automatización y visualización de los consumos presentes en el hogar, con el objetivo de que los usuarios puedan conocer los puntos más incidentes en el gasto, mostrar un perfil de consumo y controlar su activación y desactivación. Esto da como resultado un sistema de software y hardware libre que muestra que las aplicaciones manejadas con las herramientas actuales son elementos potenciales para la gestión y el cuidado del ambiente, reduciendo sustancialmente el costo de los servicios y fomentando el manejo de herramientas y soluciones económicas como el software y el hardware libre. En la actualidad, la gestión de recursos domiciliarios es uno de los temas más importantes a la hora de buscar sostenibilidad y eficiencia energética, por lo que la utilización de hardware y software libre permiten el desarrollo de aplicaciones IOT (Internet of Things), fáciles de trabajar, económicas y confiables. Finalmente, se muestran los resultados obtenidos con la medición de los consumos desde el software libre de Arduino soportado en el hardware libre de NODEMCU.

Palabras clave: eficiencia energética, sostenibilidad, hogares inteligentes, electrónica, software libre.

\section{ABSTRACT}

This article shows the creation and use of a monitoring and management system for water and energy home resources. This system allows the automation and visualization of home resources consumptions so that users can identify which are the most relevant points that generate expenses, showing consumption profiles and allowing their activation and deactivation to be controlled. This development results in a free software and hardware system, demonstrating that the use of applications handled with current tools are potential elements for the management and care of the environment, substantially reducing the cost of public utilities and fostering the use of tools and economic solutions such as free software and hardware. The management of home resources 
is currently one of the most important issues when it comes to sustainability and energy efficiency. Therefore, the use of hardware and free software allow developing Internet of Things (IOT) applications that are easy to handle, economic and reliable. Finally, we present the results obtained with the measurement of consumptions using Arduino free software with the support of NODEMCU free hardware.

Keywords: Energy efficiency, sustainability, smart homes, electronic, free software.

\section{INTRODUCCIÓN}

La domótica y las aplicaciones en Internet of Things (IOT) son soluciones en la electrónica, el software y el hardware libre que se han venido desarrollando, permitiendo que las personas tengan mayores facilidades en el desarrollo de programas y aplicaciones de software de forma gratuita y contribuyendo además a la protección del ambiente. Este tipo de desarrollos han permitido al ser humano interactuar y percibir -con la ayuda de diferentes sistemas, sensores y transductores- el comportamiento de cada variable, posibilitando un mayor control de consumos y la automatización de cada gasto. Gracias a la diversificación del software y, en especial, a la gran cantidad de software libre disponible en la actualidad, es posible generar una mayor cantidad de soluciones, como la que se plantea en este artículo. Estos sistemas evitan que se generen altos costos por la necesidad de comprar y/o adquirir software licenciado, hecho que no permite a los usuarios enfocarse en el desarrollo sino en los costos asociados a la utilización de software que requiere ser licenciado.

Para la utilización y generación del proyecto de gestión de energía y agua en el hogar se utiliza el software libre de la placa Arduino, el cual se denomina "arduino-nightly" y puede ser descargado de forma gratuita en la página directa del desarrollador (https://www.arduino.cc/). Este programa es fácil de utilizar y tiene diferentes características para el desarrollo de aplicaciones; presenta la característica de ser Open Source y un entorno de desarrollo integrado (Integrated Development Environment, IDE) de fácil manejo para los diferentes usuarios interesados en el de- sarrollo de aplicaciones en electrónica que quieran poner en práctica sus conocimientos sobre hardware y software.

Con base en lo anterior, la pregunta de investigación de esta propuesta lleva a indagar ¿Cómo pueden los sistemas electrónicos diagnosticar y medir el consumo de energía y agua en el hogar, fomentando la sostenibilidad y la educación en el cuidado de los recursos naturales y permitiendo al usuario conocer, controlar y reducir los gastos de sus servicios en tiempo real con herramientas de software libre que generen un prototipo económico?

Para resumir, el alcance del proyecto y la aplicación de software realizado buscan diagnosticar y medir los consumos en tiempo real, facilitando que los usuarios puedan visualizar estrategias de ahorro y cuidado del medio ambiente desde sus hogares, además de reducir los malos hábitos que se dan en las familias como consecuencia de la falta de educación en cuanto al ahorro de agua y energía. En este contexto, la tecnología se convierte en una solución a este tipo de problemas, ayudando a identificar medidas que incrementen la sostenibilidad y la eficiencia energética en el hogar (Gómez-Díaz, 2018).

\section{MATERIALES Y MÉTODOS}

Con la utilización del hardware libre Arduino uno (figura 1) y la tarjeta NODEMCU (figura 2) se implementa el sistema del hardware inicial, el cual permite la adquisición de los datos y el control de la información. El Arduino UNO es un dispositivo de hardware libre con el que se pueden desarrollar diferentes aplicaciones de hardware y se captura la señal de datos por medio de canales análogos, permitiendo detectar el voltaje y/o la corriente que circula por medio de un sensor. Este dispositivo, al igual que la tarjeta NODEMCU, es eficientes y económico, lo cual hace que el desarrollo de las aplicaciones que se generan desde el software libre de "Arduino-Nigthtly" no presente costos elevados y que estas sean confiables y prácticas. 
Figura 1. Arduino uno

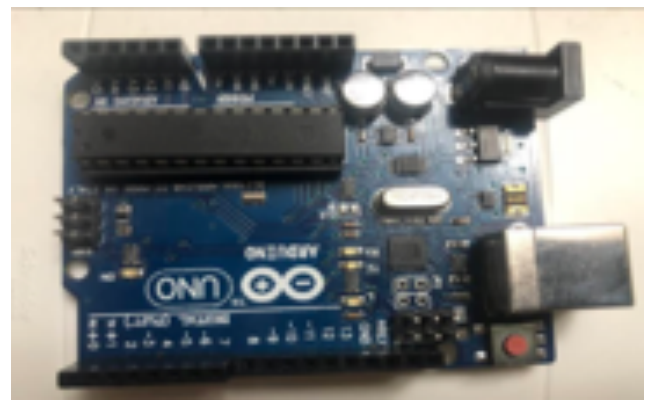

Figura 2. Tarjeta NODEMCU V3

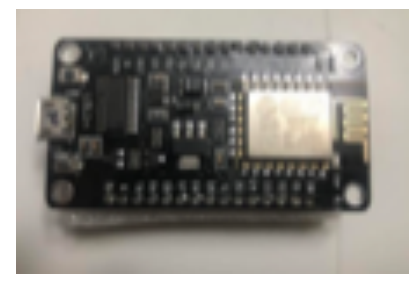

Fuente: autor

Fuente: autor

Otro de los dispositivos utilizados en el desarrollo de este proyecto es el Sensor de corriente ACS712, el cual es un elemento económico y fácil de utilizar. Este dispositivo permite, dependiendo de la referencia utilizada, dar una señal de voltaje proporcional al valor de la corriente detectada, como se muestra en la tabla 1.

Tabla 1. Características del sensor de corriente ACS712

\begin{tabular}{ccccc}
\hline Referencia del sensor & $\begin{array}{c}\text { Máxima } \\
\text { intensidad }\end{array}$ & Sensibilidad & Tensión salida & Resolución \\
\hline ACS712 05a & $\pm 5 \mathrm{~A}$ & $185 \mathrm{mV} / \mathrm{A}$ & $1,575 \mathrm{~V}-3,425 \mathrm{~V}$ & $26 \mathrm{~mA}$ \\
\hline ACS712 20a & $\pm 20 \mathrm{~A}$ & $100 \mathrm{mV} / \mathrm{A}$ & $0,5 \mathrm{~V}-4,5 \mathrm{~V}$ & $49 \mathrm{~mA}$ \\
\hline ACS712 30a & $\pm 30 \mathrm{~A}$ & $66 \mathrm{mV} / \mathrm{A}$ & $0,52 \mathrm{~V}-4,48 \mathrm{~V}$ & $74 \mathrm{~mA}$ \\
\hline
\end{tabular}

Fuente: elaboración propia.

Para la medición del consumo del agua se utiliza el sensor de flujo YF-s201, el cual tiene tres cables: rojo (5-24VDC), negro (tierra) y amarillo (salida de impulsos de efecto Hall). Al contar cada pulso de la salida del sensor es posible medir el paso del fluido, dando como resultado que cada pulso es de aproximadamente 2,25 mililitros. Es pertinente mencionar que este sensor no es de alta precisión, no obstante, debido a las cantidades de agua que se utilizan en los hogares, permite que la medición sea bastante aproximada al valor real de consumo en el hogar.

Además de los anteriores dispositivos de hardware expuestos, se utilizó el software libre de "Arduino-Nigthtly", el cual es un software Open source (o libre) que permite programar la plataforma de Arduino y la tarjeta NODEMCU desde diferentes fuentes, como la web o un pc, gracias a que es compatible con diferentes sistemas operativos (Windows, Linux y MAC OS). Estos atributos hacen posible que cualquier persona pueda adquirir y utilizar este software para la creación de diferentes soluciones, como la presentada en este artículo.
Desde un IDE el software libre permite la integración de software y hardware, los cuales, con la ayuda de diferentes librerías, brindan a los usuarios mayor facilidad en el desarrollo de diversos proyectos y soluciones, que van desde la incorporación de videojuegos hasta sistemas de control industriales. Actualmente, esta herramienta tiene una alta acogida y compatibilidad con casi todo tipo de sensores y actuadores, propiciando su versatilidad y extensión hacia las diferentes áreas del conocimiento. Lo que hace aún más llamativo a este desarrollo es que su lenguaje de programación está basado, principalmente, en $\mathrm{C}++$ y que puede ser implementado de igual forma para la ejecución de código de otros lenguajes, como el HTML y el JAVA, facilitando así la generación de soluciones de alta gama. Adicionalmente, estas características hacen que casi cualquier individuo que incursione en el campo de la programación y la electrónica pueda utilizarlo, favoreciendo la creación de más dispositivos y soluciones.

Para la implementación del proyecto se generó una metodología que permite la investigación de un pro- 
ceso más detallado desde la concepción de la idea, enmarcada en la propuesta de la tesis de maestría en ingeniería electrónica de la Universidad Nacional de Colombia desarrollada por Libardo Gómez-Díaz (2015), autor del presente artículo. Por lo anterior, es importante conocer los pasos requeridos para determinar las necesidades que se contemplan en el diseño y la planeación de este tipo de prototipos, en general, y permiten que cualquier proyecto pueda replicar este tipo de metodología para concebir cada vez mejores soluciones, generando así un prototipo funcional que realmente de solución a una necesidad.

En el presente trabajo se desarrolla una metodología que explica cada uno de los pasos involucrados en el desarrollo del proyecto, desde su concepción misma, los cuales corresponde a:

1. Recopilación de información actual, documentación y estado del arte: inicialmente se realizará lectura y recolección de documentos de proyectos y teorías implementadas en el área de la domótica y el internet de las cosas, además de las diferentes tecnologías existentes en el área de comunicaciones en el hogar, validación de sistemas eficientes de medición de energía y consumo de servicios.

2. Diseño del sistema de medición de energía: debido a que se requiere implementar un modelo en el hogar que permita la revisión de los parámetros básicos de medida, se tomará medidas de señales eléctricas para garantizar la selección y caracterización del sensor adecuado. Se procede así en la realización del diseño del sistema de medición que permita fácil instalación en el hogar. Es importante llevar a cabo simulaciones y establecer configuraciones requeridas para el equipo de medición, puesto que así se podrá revisar los resultados de las medidas obtenidas en la red eléctrica, pasando luego a la etapa de validación y de verificación de la seguridad y las protecciones del sistema.

3. Diseño de la comunicación entre los dispositivos eléctricos y electrónicos: se hace necesario realizar una integración para el modelo de comunicaciones del sistema probando diferentes tecnologías, como Bluetooth y wifi, tras lo cual se analiza cada una de las señales y se evalúan las ventajas y desventajas del sistema a integrar y la redundan- cia de los datos, garantizando con ello una comunicación constante y el adecuado almacenamiento de la información.

4. Diseño de la aplicación de integración de la información y datos: el sistema final de monitoreo y diagnóstico debe tener una interfaz que le permita al usuario interactuar con el hardware y el ahorro en el consumo de energía, por lo que se debe analizar y seleccionar el software más adecuado para la integración de los modelos de control realizados en la comunicación de dispositivos y la medición de variables. Dicha aplicación debe permitir al usuario final un mejor control de los servicios y de las necesidades de ahorro y de conocimiento de la energía del hogar, así como proporcionar herramientas y alertas de ahorro y gasto que contribuyan a que los usuarios ahorren agua y energía.

5. Construcción del prototipo funcional: en esta etapa se lleva a cabo la implementación de los circuitos y su conexión, así como el diseño estructural de la presentación final de operación. Adicionalmente, se verifica y se hacen pruebas finales del sistema completo.

6. Pruebas e integración del funcionamiento del sistema: para la puesta en marcha del sistema se deben realizar pruebas reales en campo, generando así una etapa validación con usuarios finales del sistema que busca garantizar la funcionalidad y seguridad del sistema.

7. Diagnóstico y evaluación de resultados: para efectos de validar las mediciones y comprobar los resultados de ahorro se selecciona un hogar, en el cual, durante un periodo de tres (3) meses, se tomarán las medidas de consumo de agua y de energía con el fin de evidenciar los niveles de ahorro generados al tener el sistema activado y compararlos con los niveles registrados con el sistema inactivo.

8. Documentación y resultados finales: para el paso final, se realiza la selección y explicación de las pruebas y las investigaciones realizadas, así como de las recomendaciones y las propuestas de mejora que se presenten en este proyecto. 
Figura 3. Resumen de la metodología

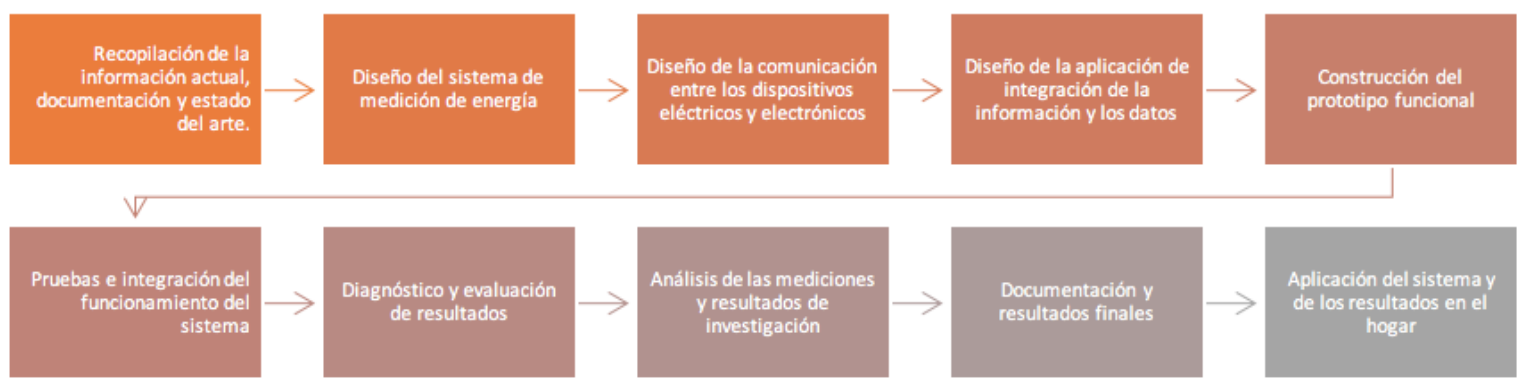

Fuente: elaboración propia.

\section{RESULTADOS}

Como resultado principal se presenta un sistema de gestión y monitoreo electrónico del consumo de agua y energía en el hogar, el cual -gracias al desarrollo realizado en el software Arduino Nigthtly- incorpora un hardware y un software que, en conjunto, facilitan la medición del consumo de energía en cada uno de los dispositivos del hogar y permiten determinar los perfiles de consumo diarios, así como determinar las horas críticas y las fugas de energía presentes en el hogar de los usuarios. Dicho programa se soporta con HTML desde el código generado del Arduino y envía los datos por wifi para que sean almacenados en una base de datos en PhpmyAdmin, lo que permite luego obtener la gráfica del consumo, tal como se visualiza en la figura 4. Lo anterior le ayuda al usuario a identificar sus consumos y compararlos con los que se reportan en el recibo de energía, así como evidenciar las fugas de energía o los puntos de gestión que requieren de la implementación de mejoras.

Figura 4. Visualización del programa cargado en HTML desde el NODEMCU

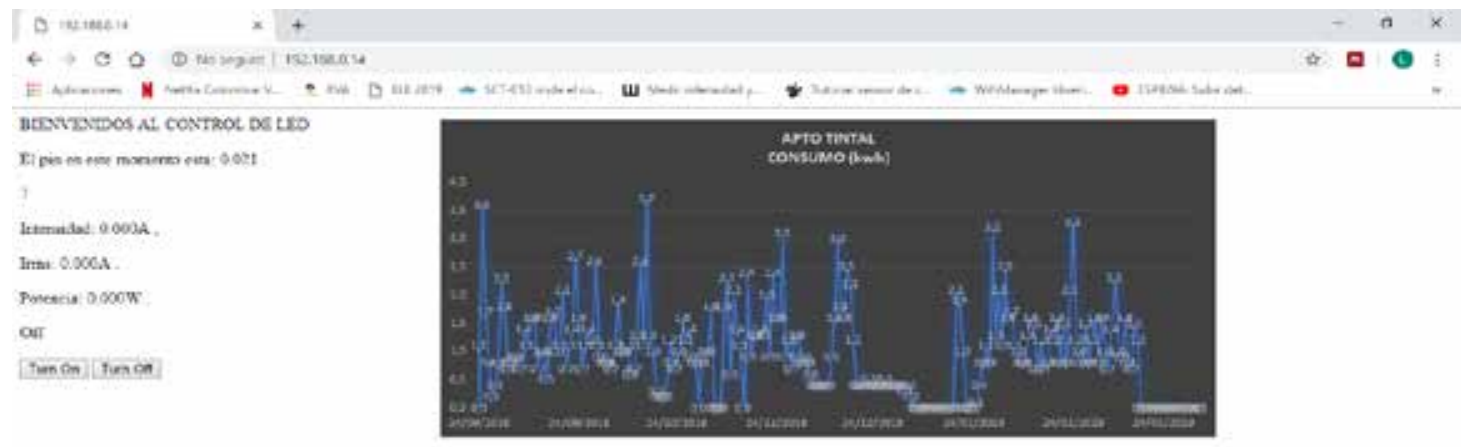

La figura 4 permite apreciar el software realizado desde software libre, donde la red permite que los usuarios puedan monitorear fácilmente los consumos que se han realizado diariamente por medio de la gráfica de datos tomada desde el NODEMCU con valores previos. El sistema automático, de igual forma, maneja alertas y avisos con mensajes para el usuario, los que le permiten educarse y conocer los límites de consumo y le informan sobre el exceso de consumo, tanto de agua como de energía, dando de esta forma una garantía de la "sostenibilidad inductiva" y propiciando un hogar más efic iente, con una mayor gestión y planificación de gastos. 
Antes de implementar el sistema se presentan altos consumos que el usuario no puede controlar y que le generan gastos; además, el usuario ni siquiera conoce los valores de consumo en tiempo real. En la figura 5 se representan los consumos más relevantes de ener- gía medidos sin la implementación del sistema en una pequeña tabla. De igual forma, en esta figura se puede evidenciar cuáles son los electrodomésticos que más generan gastos de energía en el hogar (representados en términos de porcentaje).

Figura 5. Resultados de consumo antes de la implementación.

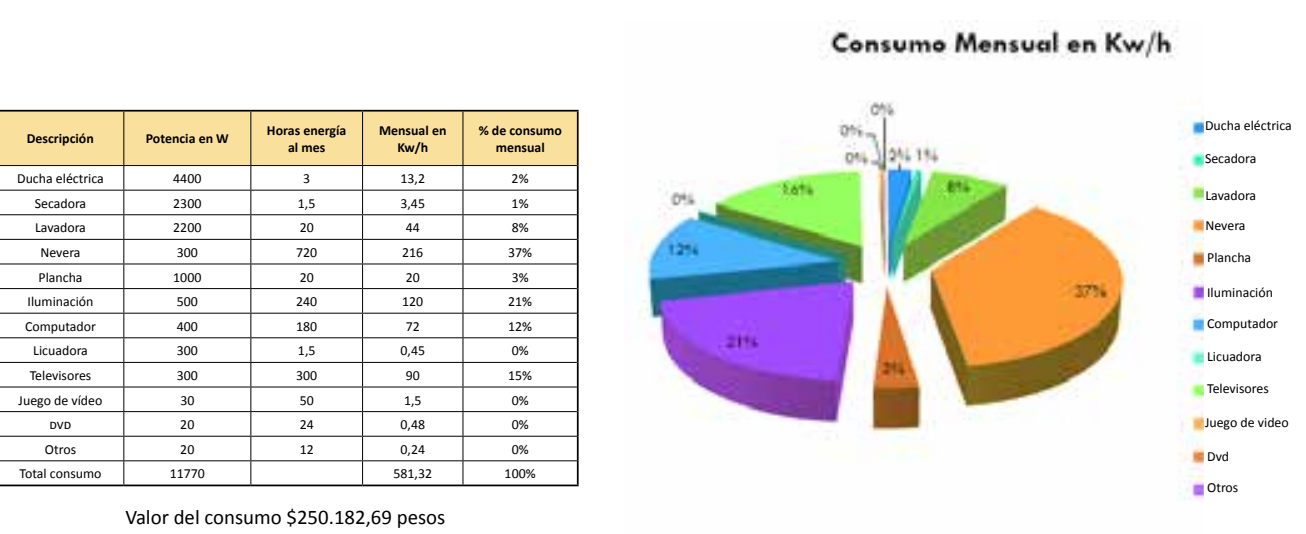

Fuente: elaboración propia.

Los resultados obtenidos tras la implementación del modelo de ahorro y gestión por aproximadamente 3 meses de prueba inicial, evidencian ahorros de 26,8 y $11,4 \%$ en el consumo de energía y agua, respectivamente, para un hogar constituido por 5 personas ( 3 adultos y 2 niños). Es de mencionar que los datos de consumo son registrados en una base de datos que -con la ayuda del NODEMCU- es enviada por wifi para garantizar su correcto almacenamiento.
Estos hallazgos permiten que el usuario tenga mayor conocimiento de sus gastos e influyen directamente en la disminución del consumo a través del envío de mensajes y alertas, evitando que se genere un mayor gasto en los recursos domiciliarios y, en este caso, disminuyendo los tiempos de energización de los diferentes elementos eléctricos y electrónicos que producen los integrantes de la familia. La figura 6 muestra los consumos obtenidos luego de la implementación del sistema, dando cuenta del ahorro obtenido en términos de energía y de dinero.

Figura 6. Resultados de consumo y ahorro después de la implementación
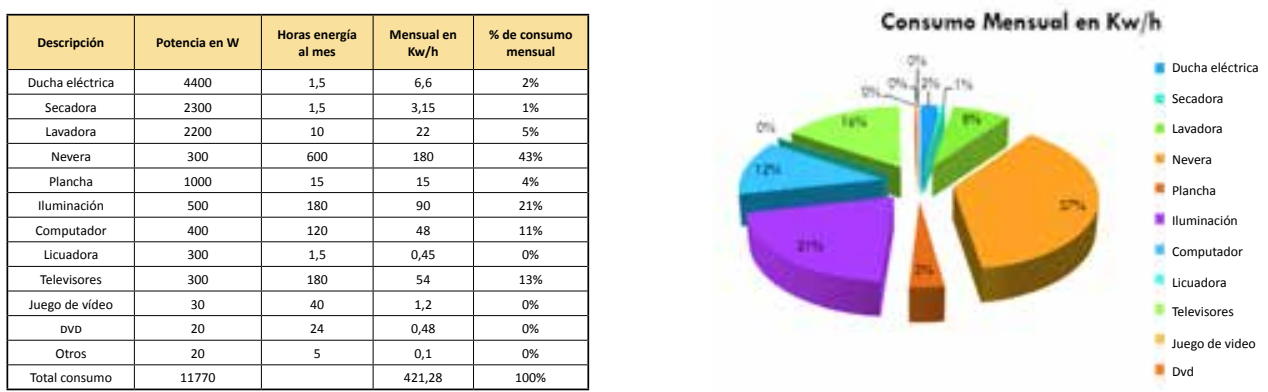

Valor del consumo $\$ 181.306,27$ pesos

Ahorro por $\$ 68.876,41$ pesos Reducción de 27,530\% 
Inicialmente, las pruebas se realizaron dentro de un apartamento que cuenta con 3 habitaciones, una sala-comedor, 2 baños y una cocina. Se realizó la conexión de los sistemas de hardware según se muestra en la figura 7, donde se evidencia la conexión para la medida de energía eléctrica consumida en el hogar (este montaje se encuentra actualmente en medición de consumo de energía). La conexión para medición de consumo de agua se encuentra en evaluación inicial, pero se implementará en nuevas investigaciones con el montaje presentado en la figura 8 , el cual cuenta con la conexión entre el NODEMCU y el ESP8266 y el sensor de flujo por efecto Hall YF-S201.

Figura 7. Prototipo de circuito para la medición de la cantidad de energía consumida
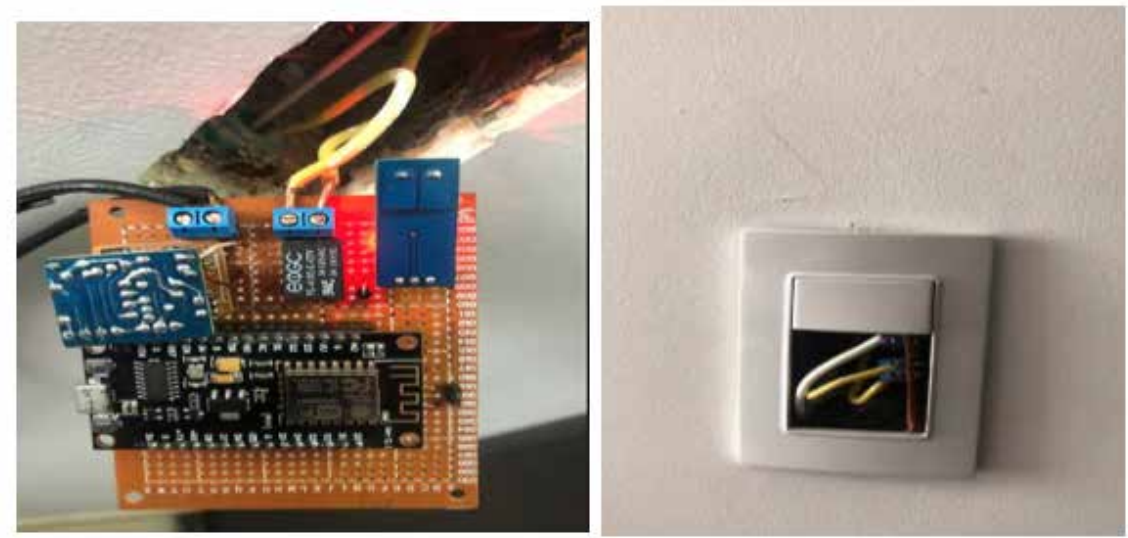

Fuente: autor.

Figura 8. Prototipo de circuito para la medición de la cantidad de agua consumida y sensor de flujo

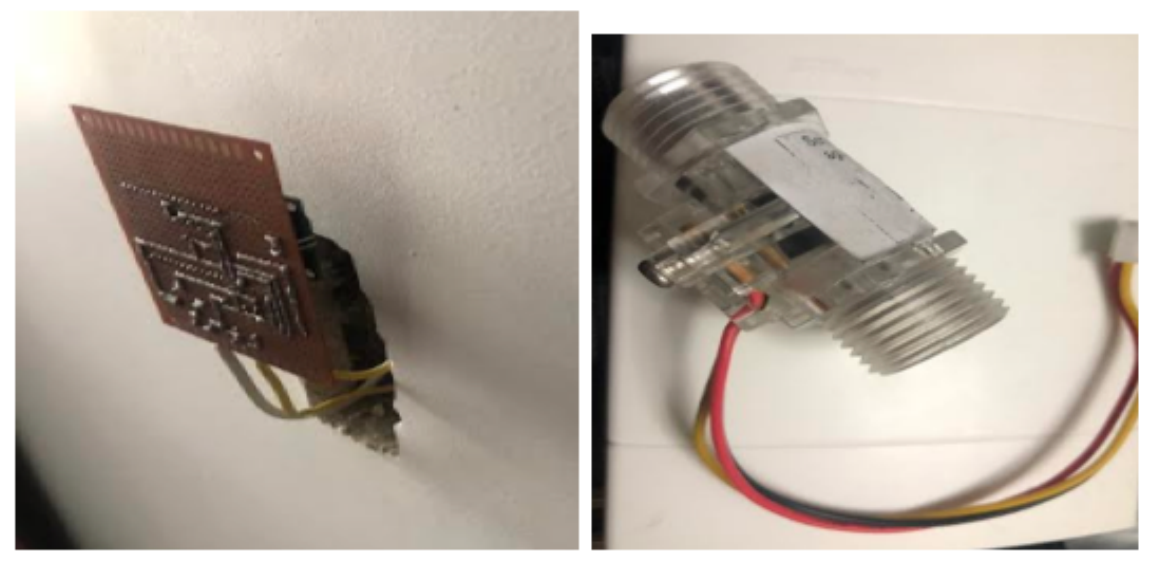

Fuente: autor.

Otro aporte de esta investigación es su contribución a la sostenibilidad inductiva, dado que el concepto busca que desde el sistema, con la ayuda de las alarmas configuradas, los usuarios puedan interactuar directamente con el ahorro y que reciban mensajes auditivos que les ayuden a mantener buenas prácticas de bajo consumo. Como se ha evidenciado en los resultados, se obtuvo un ahorro de $26,8 \%$ en el consumo de energía. Sin embargo, esto no se logra tan solo con la implementación del sistema, sino que es posible gracias a que el usuario adopta cada una de las recomendaciones y gestiona el gasto, permitiendo incentivar la pedagogía del cuidado del medio ambiente y de los recursos del hogar.

Por otra parte, se realizaron las mediciones correspondientes, dando como resultado un consumo total de $55 \mathrm{Kw} / \mathrm{h}$ aproximadamente, lo que equivale a $\$$ 
24.000 mensuales de consumo de energía; cifra que evidencia un alto consumo para 1 persona por un periodo de 1 mes. Con la implementación del sistema y la utilización del software de gestión, se realizó una medición por el mismo periodo con el fin de verificar el ahorro. Debido a la activa gestión del ahorro se encontró que los periodos de mayor consumo de energía son los fines de semana y que el equipo que genera un mayor consumo de energía en el hogar es la nevera. Teniendo esto presente, se reportó para el mes de estudio un consumo de $38 \mathrm{Kw} / \mathrm{h}$, el cual correspondiente a una reducción de 30,909 \% con respecto al consumo anterior.

Lo anterior da completa validez a los resultados esperados, puesto que la implementación del sistema le permitió al usuario estar más atento a sus consumos y validar fácilmente qué equipos del hogar le generan mayores gastos de energía. Como respaldo al sistema, una casa donde viven 6 personas, cuyo consumo mensual es de $581 \mathrm{Kw} / \mathrm{h}$, aproximadamente, equivalente a un costo mensual de $\$ 250.182,69$ pesos, presentó una reducción de $27,53 \%$ en el costo de este servicio tras la implementación del sistema, con una cifra de facturación de $\$ 181.306,27$ pesos.

\section{DISCUSIÓN}

Con respecto a las pruebas que se han realizado al encontrar las mediciones y el desarrollo del presente software, se puede evidenciar que este sistema presenta gran similitud con los sistemas de medida industriales actuales, los cuales muestran los consumos que se presentan en las plantas de producción y permiten una revisión constante de los consumos de energía de las diferentes maquinas. De esta manera, se puede trasladar este proyecto para la disminución del consumo al tener una mayor supervisión y conocimiento de cómo se comporta la utilización de la energía a escala industrial. Así, el sistema es comparable con el de la industria, aunque más económico, debido a la no utilización de licencias.

Por lo anterior, es importante anotar que los costos del consumo de energía industrial y los consumos en el hogar no son comparables, a menos que se analice en una gran cantidad de viviendas al tiempo, debido a que los ahorros del hogar pueden superar el $31 \%$. Según los resultados obtenidos, entre mayor sea la cantidad de viviendas que se incluyan en el ahorro mayor será el valor y la cantidad de ahorro generados, logrando así que pueda llegar a ser comparable el consumo de las industrias con los ahorros que se pueden lograr en barrios completos o ciudades completas, mostrando además que la electrónica, los programas y/o las aplicaciones de software, son una solución más a la sostenibilidad ambiental, y no los causantes de problemas de contaminación y generación de desechos

El desarrollo de tecnologías como el internet de las cosas permite que la información se convierta en un medio para que los seres humanos conozcan y tengan una mayor gestión e interacción con los procesos que ejecutan día a día, siendo la información acerca de los consumos del hogar los elementos que facilitan este proyecto, cuyo objetivo es que desde el hogar se inicie la gestión del cuidado y ahorro en el consumo de energía y agua, permitiendo además estar más atentos a los cobros que realizan las empresas de servicios públicos y, de igual forma, a los cuidados necesarios para la sostenibilidad del planeta.

El costo del proyecto finalmente depende de cuántos sistemas se desee monitorear en el hogar y del tamaño de este. No obstante, lo más importante radica en que el retorno a la inversión se dará de manera más rápida en tanto el usuario tenga una mayor consciencia frente el consumo de recursos y el ahorro proveniente de la adopción de las mejores prácticas de consumo.

\section{CONCLUSIONES}

Este tipo de soluciones de software han contribuido no solo en el desarrollo del sistema de gestión mismo, sino que, gracias a su naturaleza de software libre, la realización de este tipo de sistemas no involucra grandes costos asociados al desarrollo de la aplicación, puesto que no se requiere la compra de programas ni la adquisición de permisos o licencias.

Se debe tener en cuenta que la sostenibilidad inductiva es un concepto nuevo adoptado por este proyecto de investigación, el cual depende en gran medida del usuario final, considerando que se busca brindar pautas y generar una pedagogía a dicho usuario para que de forma inductiva se le recomienden mejores formas de ahorro. Esto hace que, poco a poco, se fomente en el usuario una mejor planificación de los gastos, 
contribuyendo a que se convierta en un participante activo del cuidado no solo de su presupuesto, sino también de la naturaleza.

Con respecto a los resultados, es importante anotar que la aplicación de software para este proyecto puede ser mejorada con el fin de que el usuario final pueda agregar diferentes dispositivos, sin importar las tecnologías, logrando que el desarrollo tenga una mejor acogida. Por ende, este software no solo debe ser diseñado para herramientas de computador, sino que debe incorporar su utilización desde la web, permitiendo la implementación desde un sistema operativo diferente que se pueda desarrollar en compatibilidad con todos, tanto en software como en hardware.

Gracias a los resultados obtenidos se puede evidenciar que la utilización de hardware y software libre permite que los usuarios puedan ser agentes activos del cuidado de los recursos en el hogar, así como de su planificación y educación en hábitos de ahorro, lo que hace factible que se implementen soluciones prácticas para el ahorro de energía y el cuidado del ambiente en los hogares.

\section{REFERENCIAS}

Brown, K. (2014). Build a cloud-ready temperature sensor with the Arduino Uno and the IBM Watson IoT Platform, Part 1, Build the circuit and set up the environment. Disponible en https://www.ibm. com/developerworks/cloud/library/cl-bluemixarduino-iot1/index.html

Cabrera, J., Mena, M., Parra, A., \& Pinos, E. (2016). Intelligentassistanttocontrol homepowernetwork. En 2016 IEEE International Autumn Meeting on Power, Electronics and Computing, ROPEC. https:// doi.org/10.1109/ROPEC.2016.7830531

Cruz-Ardila, J. C., Cardona-Gómez, J. C., \& HernándezPorras, D. M. (2013). Aplicación electrónica para el ahorro de energía eléctrica utilizando una energía alternativa. Entramado, 9(2), 234-248.

Gómez-Díaz, L. (2018). Propuesta de tesis de maestría en la Universidad Nacional de Colombia en diseño de sistema de gestión y monitoreo de consumo de servicios domiciliarios. Universidad Nacional de Colombia, Bogotá.
Gómez-Díaz, L. (2019). Prototipo paraimplementación de sistema de gestión y monitoreo de consumo de servicios domiciliarios. METALNNOVA SENA, 1, 10-16.

Guacaneme, G., \& Pardo, D. (2016). Diseño e implementación de un sistema de medición de consumo de energía eléctrica y agua potable remoto con interacción al usuario basado en el concepto "internet de las cosas". Tesis de pregrado. Universidad Distrital Francisco José de Caldas, Bogotá.

Halvorsen, H. P. (2014). Introduction of Arduino an Open-Source Prototyping Platform. University College of Southeast Norway.

Huérfano, P. A. (2011). Estudio para reducción de consumo energético en Colombia Basado en Tecnología Domótica. Tesis de pregrado. Facultad Ingenierías, Fundación Universitaria Konrad Lorenz, Bogotá.

Kaneko, M., Arima, K., Murakami, T., Isshiki, M., \& Sugimura, H. (2017). Design and implementation of interactive control system for smart houses. En 2017 IEEE International Conference on Consumer Electronics, ICCE. https://doi.org/10.1109/ ICCE.2017.7889320

Khajenasiri, I., Estebsari, A., Verhelst, M., \& Gielen, G. (2017). A Review on Internet of Things Solutions for Intelligent Energy Control in Buildings for Smart City Applications. Energy Procedia, 111, 770-779. https://doi.org/10.1016/j.egypro.2017.03.239

Mbarek, B., Meddeb, A., Ben, J. W., \& Mosbah, M. (2017). A secure electric energy management in smart home. International Journal of Communication Systems, 30(17), e3347. https:// doi.org/10.1002/dac.3347

Quintana, B., Pereira-Poveda, R., \& Vega, C. (2015). Automatización en el Hogar: un proceso de diseño para viviendas de Interés social. Revista EAN, 78, 108-121,.https://d oi.org/10.21158/01208160. n78.2015.1193

Salman, L., Salman, S., Jahangirian, S., Abraham, M., German, F., Blair, C., \& Krenz, P. Energy efficient loTbased smart home. En 2016 IEEE 3rd World Forum 
on Internet of Things, https://doi.org/10.1109/

WF-IoT.2016.7845449

Vega, A. M., Santamaría, F., \& Rivas, E. (2014). Internet de los Objetos empleando Arduino para la gestión eléctrica Domiciliaria. Revista EAN, 77, 22-41. 